\title{
Order parameter description of walk-off effect on pattern selection in degenerate optical parametric oscillators
}

\author{
Majid Taki \\ Laboratoire de Physique des Laseres, Atomes et Molécules, CNRS UMR 8523, Centre d'Études et de Recherches Lasers et Applications, \\ Université des Sciences et Technologies de Lille, UFR de Physique Bât. P5, F-59655 Villeneuve d'Ascq Cedex, France \\ Maxi San Miguel and Marco Santagiustina \\ Instituto Mediterráneo de Estudios Avanzados, IMEDEA (CSIC-UIB), * E-07071 Palma de Mallorca, Spain
}

(Received 10 September 1999)

\begin{abstract}
Degenerate optical parametric oscillators can exhibit both uniformly translating fronts and nonuniformly translating envelope fronts under the walk-off effect. The nonlinear dynamics near threshold is shown to be described by a real convective Swift-Hohenberg equation, which provides the main characteristics of the walk-off effect on pattern selection. The predictions of the selected wave vector and the absolute instability threshold are in very good quantitative agreement with numerical solutions found from the equations describing the optical parametric oscillator.
\end{abstract}

PACS number(s): 42.65.Tg, 46.65.Sf, 42.50.-p, 42.65.Yj

In the last few years, the interest in pattern formation in nonlinear optics has increased considerably [1]. This stems from the fact that nonlinear optical systems show the complexity of other spatially extended systems [2] in a context that presents additional peculiarities such as the light polarization [3] and the macroscopic manifestations of quantum properties [4]. Among all the possible devices, optical parametric oscillators (OPO's) have recently appeared as one of the most promising, not only for the richness in nonlinear dynamics [5] but also for their potential applications [6], including low-noise measurements and detection [7] and the possible storage of information [8,9].

In OPO's, the nonlinear quadratic down-conversion process is most efficient when the phase matching condition is fulfilled. This can be achieved by taking advantage of the crystal birefringence to compensate for the unavoidable material chromatic dispersion. As a result ordinary and extraordinary rays may have different Poynting vector directions and therefore the rays may diverge, i.e., walk-off, from one another as they propagate through the nonlinear medium. Very recently, convective instabilities and noise-sustained patterns have been shown to appear due to the walk-off $[10,11]$. For degenerate (D) OPO's, an interesting recent result [12] is the continuous generation of patterns of dark solitary waves [13] for positive signal detuning (where only a homogeneous state can develop in the absence of walkoff). The pattern is created behind a propagating front leading to nonuniformly translating envelope fronts. However, for DOPO's, the authors of Ref. [11] predicted, based on an amplitude equation description, that only uniformly translating fronts (where no pattern can be formed) exist for large finite positive signal detunings. Therefore, DOPO's exhibit both types of fronts and a universal description of the walkoff effect on pattern selection is worth addressing.

In this paper we present a theoretical study of the dynamical transition from uniformly translating fronts, leading be-

\footnotetext{
*http;//www.imedea.uib.es/PhysDept
}

hind them a homogeneous state, to nonuniformly translating envelope fronts, giving rise to the formation of a nonlinear pattern. The exact analytical expression of the transition curve in terms of signal detuning and walk-off parameters is found. It reveals the existence of a scaling law governing the transition. Using this scaling we have performed an order parameter description, near threshold, which leads to a real convective Swift-Hohenberg equation. This equation provides the generic description of the walk-off effect on transverse pattern formation. Based on this reduced model we have obtained analytical expressions for the wave-vector selection rules and the threshold of the absolute instability. The predictions are in very good agreement, both qualitatively and quantitatively, with numerical simulations of the DOPO's original equations.

We start from the standard model of a type I phasematched degenerate OPO in the mean-field approximation $[10,11]$ :

$$
\begin{gathered}
\partial_{t} A_{0}=\gamma_{0}\left[-\left(1+i \Delta_{0}\right) A_{0}+i a_{0} \triangle_{\perp} A_{0}-A_{1}^{2}+E(x, y)\right], \\
\partial_{t} A_{1}=\gamma_{1}\left[-\left(1+i \Delta_{1}\right) A_{1}+i a_{1} \triangle_{\perp} A_{1}+A_{0} A_{1}^{*}+\rho_{1} \partial_{y} A_{1}\right],
\end{gathered}
$$

where $A_{0}$ and $A_{1}$ are the normalized slowly varying envelopes for pump and signal fields, respectively. The parameters $\Delta_{0,1}, \gamma_{0,1}$, and $a_{0,1}$ are the detunings, the cavity decay rates, and the diffraction, respectively. The remaining parameters are the normalized external pump $E$ and the signal walk-off coefficient $\rho_{1}$. For a detailed discussion of the linear stability analysis (LSA) of the steady state $A_{0}=E_{0} /(1$ $\left.+i \Delta_{0}\right), A_{1}=0$ including the walk-off we refer to previous published papers $[10,11]$. Here we just recall that when $\rho_{1}$ $\neq 0$ the absolute instability threshold, at which a dynamically self-sustained structure can develop, is such that $\operatorname{Re}\left[\lambda\left(\mathbf{k}^{s}\right)\right]$ $=0$ where $\mathbf{k}^{s}=\left(k_{x}^{s}, k_{y}^{s}\right)$ is a complex saddle point satisfying $\nabla_{\mathbf{k}} \lambda(\mathbf{k})=\mathbf{0}$ and $\operatorname{Re}\left[\nabla_{\mathbf{k}}^{2} \lambda(\mathbf{k})\right] \geqslant 0$ where $\lambda(\mathbf{k})$ is the linearized eigenvalue $[10,11]$. The use of a complex wave vector 


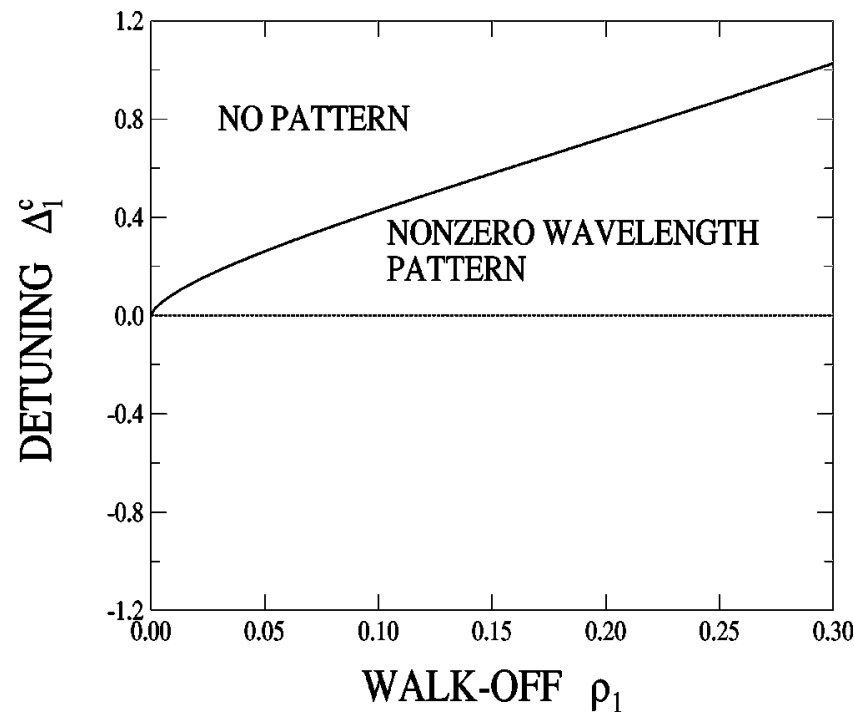

FIG. 1. Critical curve (solid line) $\Delta_{1}^{c}\left(\rho_{1}\right)$ [Eq. (5)] limiting the new region of positive signal detunings of the existence of patterns. The horizontal dashed line is the limit without walk-off. The diffraction parameter $a_{1}=0.25$.

to express the linearized solutions $\exp (\mathbf{k} \cdot \mathbf{r}+\lambda t)$ is not surprising in this context [10-12]: Its imaginary part accounts for the mode oscillations and the real part for the envelope front. The gradient condition is satisfied for $k_{x}^{s}=0$, which means that the stripes of the selected pattern are oriented orthogonally to the walk-off [10-12]. At the transition from nonzero wavelength patterns to homogeneous states, $\operatorname{Im}\left(k_{y}^{s}\right)$ vanishes, which leads to a real wave vector $\mathbf{k}^{s}=\left[k_{x}^{s}=0, k_{y}^{s}\right.$ $\left.=\operatorname{Re}\left(k_{y}^{s}\right)\right]$. This simplifies considerably the complex expression of $\lambda(\mathbf{k})$ which reduces to a real function of $\mathbf{k}$. Thus we obtain for the most unstable mode $\left(\operatorname{Re}\left[\lambda\left(\mathbf{k}^{s}\right)\right]=0\right)$ :

$$
2 a_{1}^{2}\left(k_{y}^{s}\right)^{3}-\left(2 a_{1} \Delta_{1}-\rho_{1}^{2}\right) k_{y}^{s}-\rho_{1}=0 .
$$

Real solutions $k_{y}^{s}$ are possible only if the signal detuning $\Delta_{1}$ satisfies

$$
\Delta_{1} \geqslant \Delta_{1}^{c}\left(\rho_{1}\right)=\frac{3}{2\left(2 a_{1}\right)^{1 / 3}} \rho_{1}^{2 / 3}+\frac{1}{2 a_{1}} \rho_{1}^{2} .
$$

Note the dependence of $\Delta_{1}^{c}\left(\rho_{1}\right)$ on the diffraction parameter $a_{1}$ leading to high values of critical signal detunings for small values of $a_{1}$. The critical curve at transition $\Delta_{1}=$ $\Delta_{1}^{c}\left(\rho_{1}\right)$ is shown in Fig. 1.

Since the walk-off parameter $\rho_{1}$ is small, the above relation shows that pattern formation is possible only for small detunings, i.e., such that $\Delta_{1}<\Delta_{1}^{c}\left(\rho_{1}\right)$. This is in agreement with the fact that for $\Delta_{1}$ of order unity only nonlinear homogeneous states can develop. In this case nonlinear dynamics is well described by a real Ginzburg-Landau equation (GLE) [11]. However, it is clear that GLE fails in the parameter range of pattern formation. A universal description, valid in both regimes, can be derived (for $a_{1}$ fixed) from the scaling law $\Delta_{1}^{c}\left(\rho_{1}\right) \sim \rho_{1}^{2 / 3}$. To this purpose, we have performed a weakly nonlinear analysis of the full DOPO equations (1) around threshold for signal generation by setting $\Delta_{1}=\varepsilon \Delta$, which leads to $\rho_{1}=\varepsilon^{2 / 3} \rho$ with $\Delta, \rho$ of order one. The small parameter $\varepsilon$ measures the distance from threshold $\left(\mu^{2}-1\right.$ $\left.=\varepsilon^{2}\right)$ in the order parameter description approach developed in a previous work [11]. The evolution equations for the field amplitude variables are obtained by expanding the solution $\left(A_{0}, A_{1}\right)$ in power series of $\varepsilon:\left(A_{0}, A_{1}\right)=\varepsilon \quad\left(\psi_{0}, \psi_{1}\right)$ $+\varepsilon^{2}\left(\psi_{0}^{(2)}, \psi_{1}^{(2)}\right)+\cdots$.

With these scalings the following convective SwiftHohenberg equation (SHE) is obtained for the signal:

$$
\begin{aligned}
\partial_{\tau} \psi_{1}= & \frac{1}{2}\left(\mu^{2}-1\right) \psi_{1}-\frac{1}{2}\left(\Delta_{1}-a_{1} \triangle_{\perp}^{2}\right)^{2} \psi_{1} \\
& +\rho_{1} \partial_{y} \psi_{1}-\psi_{1}^{3} /\left(1+\Delta_{0}^{2}\right)
\end{aligned}
$$

where we have set $\tau=\gamma_{1} t$ and $\psi_{1}$ is a real function as a consequence of the fact that the OPO is degenerate. The description of DOPO's in terms of a SHE was also the result of Refs. $[14,15]$; here we also account for the walk-off with the correct scaling relations presented above. Our main aim is to examine the emerging dynamically self-sustained patterns so that we restrict the analytical study to the occurrence of the absolute instability for which the linearization of the convective SHE around $\psi_{1}=0$ gives

$$
\lambda(\mathbf{k})=\frac{1}{2}\left(\mu^{2}-1\right)-\frac{1}{2}\left(\Delta_{1}-a_{1} k^{2}\right)^{2}+\rho_{1} k_{y},
$$

where $\mathbf{k}$ is a complex wave vector satisfying the saddle point condition,

$$
\begin{gathered}
\frac{\partial \lambda}{\partial k_{x}}=2 a_{1}\left(\Delta_{1}-a_{1} k^{2}\right) k_{x}=0, \\
\frac{\partial \lambda}{\partial k_{y}}=2 a_{1}\left(\Delta_{1}-a_{1} k^{2}\right) k_{y}+\rho_{1}=0 .
\end{gathered}
$$

From the system (7) we recover the condition $k_{x}^{s}=0$ while the $k_{y}^{s}$ component satisfies:

$$
2 a_{1}^{2}\left(k_{y}^{s}\right)^{3}-2 a_{1} \Delta_{1}\left(k_{y}^{s}\right)-\rho_{1}=0 .
$$

Note that this equation is more tractable mathematically than the original one and it also allows one to recover the transition curve $\Delta_{1}^{c}\left(\rho_{1}\right)$ to the leading order $\rho_{1}$. From Eq. (8), the formation of patterns corresponding to nonuniformly translating envelope fronts is possible if $\Delta_{1}<\Delta_{1}^{c}\left(\rho_{1}\right)$ and their wave vectors are

$$
\begin{aligned}
& \operatorname{Im}\left(k_{y}^{s}\right)=\frac{\sqrt{3}}{2}\left(\frac{\rho_{1}}{4 a_{1}^{2}}\right)^{1 / 3}\left[\xi_{+}^{1 / 3}-\xi_{-}^{1 / 3}\right], \\
& \operatorname{Re}\left(k_{y}^{s}\right)=-\frac{1}{2}\left(\frac{\rho_{1}}{4 a_{1}^{2}}\right)^{1 / 3}\left[\xi_{+}^{1 / 3}+\xi_{-}^{1 / 3}\right],
\end{aligned}
$$

where $\xi_{ \pm}=1 \pm \sqrt{1-\left(\Delta_{1} / \Delta_{1}^{c}\right)^{3}}$. The most unstable modes are those for which $\operatorname{Re}\left[\lambda\left(k_{y}^{s}\right)\right]=0$ and they oscillate with frequency $\operatorname{Im}\left[\lambda\left(k_{y}^{s}\right)\right]$. Since they drift at velocity $\rho_{1}$ and, as long as no nodes disappear or are created in the nonlinear region behind the front, the conservation of the linear flux of 


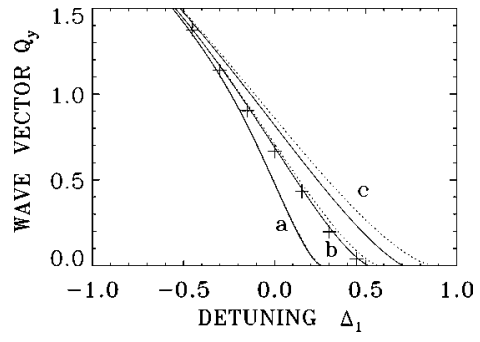

FIG. 2. The solid curves are the predicted wave vectors [Eq. (11)] of the emerging signal pattern from the convective SHE for (a) $\rho_{1}=0.05$, (b) $\rho_{1}=0.15$, (c) $\rho_{1}=0.25$. The dotted curves are the corresponding numerical solutions from the LSA. The crosses represent the wave vectors selected in the numerical solutions of the governing equations (1). The remaining parameters are set to $\Delta_{0}$ $=0, \gamma_{1}=\gamma_{0}=1$, and $a_{0}=0.125$.

the field nodes can be applied [12], the wave number of the generated pattern is then defined as

$$
\begin{aligned}
Q_{y} \equiv & \operatorname{Im}\left[\lambda\left(k_{y}^{s}\right)\right] / \rho_{1} \\
= & \operatorname{Im}\left(k_{y}^{s}\right)\left\{1+2 a_{1} \rho_{1}^{-1} \operatorname{Re}\left(k_{y}^{s}\right)\right. \\
& \left.\times\left[\Delta_{1}-a_{1}\left(\operatorname{Re}\left(k_{y}^{s}\right)^{2}-\operatorname{Im}\left(k_{y}^{s}\right)^{2}\right)\right]\right\} .
\end{aligned}
$$

Notice that, apart from the case of uniformly translating fronts $\left[\operatorname{Im}\left(k_{y}^{s}\right)=Q_{y}=0\right], \operatorname{Im}\left(k_{y}^{s}\right)$ and $Q_{y}$ are generally different. The predicted wave number $Q_{y}$ as a function of the signal detuning is reproduced in Fig. 2 together with the wave number selected in the exact numerical solutions of Eq. (1) and that predicted by the LSA of the same equations. As can be seen from the figure the agreement between the analytical predictions and the exact, numerically found values is excellent, especially for small $\rho_{1}$ and $\Delta_{1}$.

The threshold of absolute instability can be calculated analytically from the condition $\operatorname{Re}\left[\lambda\left(k_{y}^{s}\right)\right]=0$ for both types of fronts. After straightforward calculations we obtain

$$
\mu_{A}^{2}=\left\{\begin{array}{r}
1+4 a_{1}^{2} \operatorname{Re}\left(k_{y}^{s}\right)^{2}\left[3 \operatorname{Re}\left(k_{y}^{s}\right)^{2}+\operatorname{Im}\left(k_{y}^{s}\right)^{2}\right] \\
\text { for } \operatorname{Im}\left(k_{y}^{s}\right) \neq 0, \\
1+\left(\Delta_{1}-a_{1} \operatorname{Re}\left(k_{y}^{s}\right)^{2}\right)^{2}-2 \rho_{1} \operatorname{Re}\left(k_{y}^{s}\right) \\
\text { for } \operatorname{Im}\left(k_{y}^{s}\right)=0 .
\end{array}\right.
$$

A closed analytical form of the absolute threshold as a function of parameters can be obtained by substituting $\operatorname{Im}\left(k_{y}^{s}\right)$ and $\operatorname{Re}\left(k_{y}^{s}\right)$ by their expression in Eqs. (9), respectively. We have reproduced the absolute threshold curves

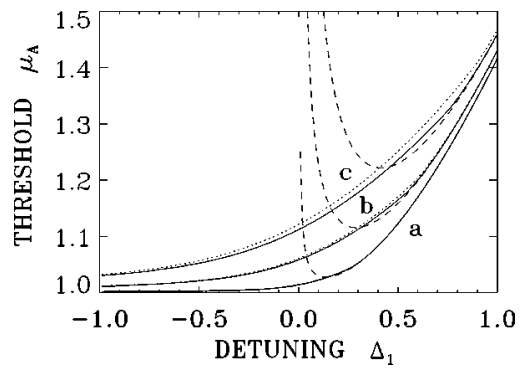

FIG. 3. Absolute threshold $\mu_{A}$ for the same values of $\rho_{1}$ as in Fig. 2. The solid curves are the values predicted by Eqs. (12), while the dotted curves are the result of the numerical solutions from the DOPO's LSA. The dashed curves are the threshold given by the GLE [11].

Eqs. (12) versus the signal detuning $\Delta_{1}$ for various $\rho_{1}$ and the curves compare very well with the corresponding numerical results from the LSA, as shown in Fig. 3. We have also showed (dashed curves) the thresholds obtained with the real Ginzburg-Landau approximation and from the figure it is clear that this approximation must be discarded in the parameter range of small signal detunings. Similar approximations for $\Delta_{1}>0$ of order unity have been proposed, leading to GLE's either without walk-off [16] or with walk-off [17]. In particular, for the DOPO, a Fisher-Kolmogorov equation (FKE, a form of the GLE with real coefficients) has been derived [17]. It is interesting to note that the FKE with a walk-off term does not show the phenomenon of pattern formation behind a propagating front studied in [12]. However, a correction of the type $\nabla^{4}$ (in the spirit of a SHE) is known to account for such a phenomenon [18].

In summary we have shown that DOPO's, in presence of walk-off, can exhibit either uniformly translating fronts or nonuniformly translating envelope fronts. The latter allows the formation of patterns even for positive detunings, giving rise to a nontrivial critical transition curve between pattern forming and homogeneous states. We also demonstrated that the critical transition curve can be obtained analytically and provides a scaling law that leads to a unified description of the dynamics through an equation of Swift-Hohenberg type. The predictions obtained with this equation, regarding the wave-vector selection and the absolute instability threshold, are compared with the numerical simulations of the original DOPO equations and the agreement is very good.

One of the authors (M.T.) acknowledges helpful discussions with P. Glorieux, N. Ouarzazi, and H. Ward. The work of M.S. was supported by the European Commission (QSTRUCT Project No. ERB FMRX-CT96-0077).
[1] Nonlinear Optical Structures, Patterns, Chaos, edited by M.S. El Naschie and L. A. Lugiato, Special issue of Chaos, Solitons Fractals 4, 1251 (1994); Pattern Formation in Nonlinear Optical Systems, edited by R. Neubecker and T. Tschudi, Special issue of Chaos, Solitons Fractals 10, 617 (1999); Proceedings of the Euroconference Patterns in Nonlinear Optical Systems, Alicante (Spain) 1998 J. Opt. B1, 1 (1999); and references therein.
[2] M. C. Cross and P. C. Hohenberg, Rev. Mod. Phys. 65, 851 (1993).

[3] M. Hoyuelos, P. Colet, M. San Miguel, and D. Walgraef, Phys. Rev. E 58, 2992 (1998), and references therein.

[4] L. A. Lugiato, M. Brambilla, and A. Gatti, Adv. At., Mol., Opt. Phys. 40, 229-306 (1999), and references therein .

[5] K. Staliunas, Opt. Commun. 91, 82 (1992); J. Mod. Opt. 42, 1261 (1995); G.-L. Oppo, M. Brambilla, and L. A. Lugiato, 
Phys. Rev. A 49, 2028 (1994); S. Longhi, ibid. 53, 4488 (1996); G. J. de Varcarcel, K. Staliunas, E. Roldan, and V. J. Sanchez-Morcillo, ibid. 54, 1609 (1996); 56, 3237 (1997).

[6] See, e.g., A. P. Piskarskas, Opt. Photonics News 8(7), 24 (1997); R. L. Byer and A. P. Piskarskas, (feature issue on optical parametric oscillators), J. Opt. Soc. Am. B 10, 1655 (1993).

[7] H. J. Kimble, in Fundamental Systems in Quantum Optics, edited by J. Dalibard, J. M. Raimond, and J. Zinn-Justine (Elsevier, Amsterdam, 1992), p. 545.

[8] M. Tlidi, P. Mandel, and M. Haelterman, Phys. Rev. E 56, 6524 (1998); S. Trillo, M. Haelterman, Opt. Lett. 23, 1514 (1998).

[9] S. Longhi, Phys. Scr. 57, 611 (1997); K. Staliunas, V.J. Sanchez-Morcillo, Phys. Rev. A 57, 1454 (1998).

[10] M. Santagiustina, P. Colet, M. San Miguel, and D. Walgraef,
Phys. Rev. E 58, 3843 (1998).

[11] H. Ward, M. N. Ouarzazi, M. Taki, and P. Glorieux, Eur. Phys. J. D 3, 275 (1998).

[12] M. Santagiustina, P. Colet, M. San Miguel, D. Walgraef, Opt. Lett. 23, 1167 (1998).

[13] S. Trillo, M. Haelterman, A. Sheppard, Opt. Lett. 22, 970 (1997).

[14] S. Longhi and A. Geraci, Phys. Rev. A 54, 4581 (1996).

[15] G. J. de Varcarcel, K. Staliunas, E. Roldan, V. J. SanchezMorcillo, Phys. Rev. A 54, 1609 (1996).

[16] Z. H. Musslimani, Physica A 249, 141 (1998).

[17] J. N. Kutz, T. Erneux, S. Trillo, and M. Haelterman, J. Opt. Soc. Am. B 16, 1936 (1999).

[18] G. Dee and J. Langer, Phys. Rev. Lett. 50, 383 (1983); G. T. Dee, W. Van Sarloos, ibid. 60, 2641 (1988). 